\title{
Mechanism of Activation of Mechanistic Target of Rapamycin Complex 1 by Methionine
}

\author{
Munehiro Kitada ${ }^{1,2 *}$, Jing $\mathrm{Xu}^{1}$, Yoshio Ogura ${ }^{1}$, Itaru Monno ${ }^{1}$ and Daisuke Koya ${ }^{1,2 *}$ \\ ${ }^{1}$ Department of Diabetology and Endocrinology, Kanazawa Medical University, Uchinada, Japan, ${ }^{2}$ Division of Anticipatory \\ Molecular Food Science and Technology, Medical Research Institute, Kanazawa Medical University, Uchinada, Japan
}

\section{OPEN ACCESS}

Edited by:

Giovanni Corsetti,

University of Brescia, Italy

Reviewed by:

Michael A. Kalwat,

University of Texas Southwestern Medical Center, United States Reinhard Christoph Dechant,

ETH Zürich, Switzerland

*Correspondence:

Munehiro Kitada

kitta@kanazawa-med.ac.jp

Daisuke Koya

koya0516@kanazawa-med.ac.jp

Specialty section:

This article was submitted to

Signaling,

a section of the journal

Frontiers in Cell and Developmental

Biology

Received: 26 May 2020

Accepted: 13 July 2020

Published: 11 August 2020

Citation:

Kitada M, Xu J, Ogura Y, Monno and Koya D (2020) Mechanism of Activation of Mechanistic Target

of Rapamycin Complex 1 by Methionine

Front. Cell Dev. Biol. 8:715 doi: 10.3389/fcell.2020.00715
Nutrients are closely involved in the regulation of lifespan and metabolic health. Cellular activities, such as the regulation of metabolism, growth, and aging, are mediated by a network of nutrients and nutrient-sensing pathways. Among the nutrient-sensing pathways, the mechanistic target of rapamycin complex 1 (mTORC1) acts as the central regulator of cellular functions, which include autophagy. Autophagy plays a significant role in the removal of protein aggregates and damaged or excess organelles, including mitochondria, to maintain intracellular homeostasis, which is involved in lifespan extension and cardiometabolic health. Moreover, dietary methionine restriction may have a beneficial effect on lifespan extension and metabolic health. In contrast, methionine may activate mTORC1 and suppress autophagy. As the mechanism of methionine sensing on mTORC1, SAMTOR was identified as a sensor of S-adenosyl methionine (SAM), a metabolite of methionine, in the cytoplasm. Conversely, methionine may activate the mTORC1 signaling pathway through the activation of phosphatase 2A (PP2A) because of increased methylation in response to intracellular SAM levels. In this review, we summarized the recent findings regarding the mechanism via which methionine activates mTORC1.

Keywords: methionine, S-adenosyl methionine, mechanistic target of rapamycin complex 1, autophagy, SAMTOR, phosphatase $2 \mathrm{~A}$ methylation

\section{INTRODUCTION}

All organisms adapt and respond to the nutrients available in the environment. Cellular activities, including the regulation of metabolism, cell growth, and aging, are mediated by a network that comprised nutrients and nutrient-sensing pathways (Efeyan et al., 2015). Dietary interventions, such as calorie or dietary restriction and protein restriction, have been widely explored for their impact on lifespan extension or the prevention of age-related diseases through effects on cardiometabolic health. Calorie or dietary restriction without malnutrition has been demonstrated to extend the lifespan of organisms and improve their cardiometabolic health (Colman et al., 2009; Fontana et al., 2010; Mattison et al., 2012, 2017). However, recent studies have reported that protein restriction, rather than calorie or dietary restriction, is more strongly involved in the lifespan extension and cardiometabolic health (Nakagawa et al., 2012; Levine et al., 2014; Solon-Biet et al., 2014; Simpson et al., 2017; Kitada et al., 2019). Moreover, accumulated evidence from experimental studies indicates that the restriction of specific amino acids, such as branched-chain amino acids (BCAAs) or methionine, promotes longevity and cardiometabolic health (Fontana et al., 2016; 
Lee et al., 2016; Cummings et al., 2018; Kitada et al., 2019), which possibly mediates the benefits of protein restriction.

Among the nutrient-sensing pathways, the mechanistic target of rapamycin complex 1 (mTORC1) is a serine/threonine protein kinase that acts as the central regulator of cell growth and metabolism in response to the changes in nutrients or growth factors (Kim and Guan, 2019). Numerous studies on the pharmacological inhibition of MTORC1 by rapamycin have demonstrated the lifespan-extension benefit of this approach (Harrison et al., 2009; Anisimov et al., 2011; Wilkinson et al., 2012; Miller et al., 2014; Zhang et al., 2014), which suggest that mTORC1 is closely involved in lifespan regulation. The mechanism via which the suppression of mTORC1 leads to lifespan extension includes the induction of the autophagy (Kim and Guan, 2019). Autophagy is a lysosomal degradation pathway that plays an important role in the removal of protein aggregates and damaged or excess organelles, such as mitochondria, to maintain homeostasis and cell function (Mizushima et al., 2008). An appropriate autophagy may protect cells against various agerelated stress conditions, which results in lifespan extension and cardiometabolic health (Wong et al., 2020). mTORC1 has been recognized as a crucial regulator of autophagy, and amino acids are one of the strong factors that affect mTORC1 activation (Kim and Guan, 2019). Thus, the beneficial effect of protein restriction on lifespan extension may be mediated through the induction of autophagy via the suppression of mTORC1 under amino-acid restriction. Recent findings have clarified that essential amino acids, including BCAAs or methionine, are possibly related to the regulation of the aging process, lifespan, and cardiometabolic health through multiple physiological and molecular mechanisms. In particular, the mechanisms underlying the role of methionine in the regulation of aging or lifespan have been widely investigated through dietary intervention via the application of a methionine restriction diet. Among these mechanisms, the involvement of methionine in the regulation of mTORC1 and autophagy has been elucidated based on the results of those studies. In the current review, we summarized the recent findings regarding the mechanism of mTORC1 activation by methionine.

\section{ROLE OF RAGS ON THE REGULATION OF MTORC1 ACTIVITY BY AMINO-ACID SENSING}

The mTORC1 activity is regulated by several molecules in response to changes in nutrients, including amino acids and growth factors. Moreover, the upstream component of the amino-acid-sensing pathway of mTORC1 is complicated (Kim and Guan, 2019). The regulation of mTORC1 activity by amino acids occurs through the translocation and localization of mTORC1 to lysosomes. The heterodimers of low-molecularweight GTPases, RagA or B, and RagC, or D (Kim et al., 2008; Sancak et al., 2008; Anandapadamanaban et al., 2019), which are localized in lysosomes, play an important role in the activation of mTORC1 by amino acids. RagA and RagC exist as a dimer, and the GTP-bound form of RagA is its active form, whereas the GDP-bound form of RagC is its active form. In the presence of amino acids, these proteins function as activated GTP-RagA or GDP-RagC. In contrast, under amino-acid starvation, they function as a combination of inactivated GDP-RagA or GTPRagC. The activated Rag dimer binds to Raptor, which is a major component of mTORC1, and participates in the translocation and localization of mTORC1 from the cytoplasm to lysosomes. Thereafter, in the lysosome, mTORC1 is activated by GTP-Rheb.

The GATOR1 and GATOR2 complexes are recognized as Rag regulators and are localized in the cytoplasm (Bar-Peled et al., 2013). GATOR1 is a complex composed of three proteins, DEPDC5, NPRL2, and NPRL3, and has RagA-binding ability and GTPase-activating protein (GAP) activity for RagA (Shen et al., 2018). DEPDC5 of GATOR1 contains a GAP domain, which binds directly to RagA, thus inactivating it. However, deletion of the GATOR1 component results in the amino-acid-independent localization and activation of mTORC1 in the lysosome, which demonstrates that GATOR1 is a negative regulator of mTORC1. In contrast, GATOR2 is a complex consisting of five proteins, Sec13, Seh1L, WDR24, WDR59, and Mios (Bar-Peled et al., 2013). GATOR2 binds to GATOR1; GATOR2 acts as the positive regulator of mTORC1 by suppressing the GAP activity of GATOR1. Leucine and arginine bind to sestrin $1 / 2$ and CASTOR1, respectively, and sestrin1/2 and CASTOR1 are also recognized as sensors of leucine or arginine (Figure 1A). Amino-acid-bound sensor proteins dissociate from GATOR2, thus losing their ability to inactivate GATOR2 (Figure 1A; Chantranupong et al., 2014, 2016; Parmigiani et al., 2014; Saxton et al., 2016a,b; Wolfson et al., 2016). Consequently, the activated GATOR2 triggers the activation of mTORC1 through the inactivation of GATOR1. Conversely, during leucine or arginine starvation, sestrin $1 / 2$ and CASTOR1 bind to GATOR2 and inactivate GATOR2, which results in mTORC1 inactivation via an increase in the RagA GAP activity of GATOR1 (Figure 1B).

In addition to the cytosolic amino-acid-sensing branch, Shen and Sabatini reported that Ragulator and SLC38A9 are two critical regulators of the activation of mTORC1 as the lysosomal amino-acid-sensing branch (Shen and Sabatini, 2018) (Figure 1C). Ragulator tethers the Rag heterodimer to the lysosomal surface, and the SLC38A9 transmembrane protein is a lysosomal arginine sensor that stimulates mTORC1 activity through the regulation of Rags. Ragulator and SLC38A9 are guanine exchange factors that lead the Rags toward the active form (Shen and Sabatini, 2018). Ragulator triggers GTP release from RagC, thus lifting the locked inactivated state of the Rags (Shen and Sabatini, 2018). Upon arginine binding, SLC38A9 converts RagA from the GDP- to the GTPbound state, leading to the activation of the Rags (Shen and Sabatini, 2018). Thus, Ragulator and SLC38A9 activate mTORC1 by recruiting it to the lysosomal surface via Rag activation in response to arginine levels in the lysosome. Moreover, v-ATPase interacts with Ragulator, Rags, and SLC38A9 and is involved both in amino-acid-sensing and in efflux from the lysosome (Zoncu et al., 2011; Abu-Remaileh et al., 2017; Wyant et al., 2017). However, it remains unknown whether v-ATPase senses amino acids. 


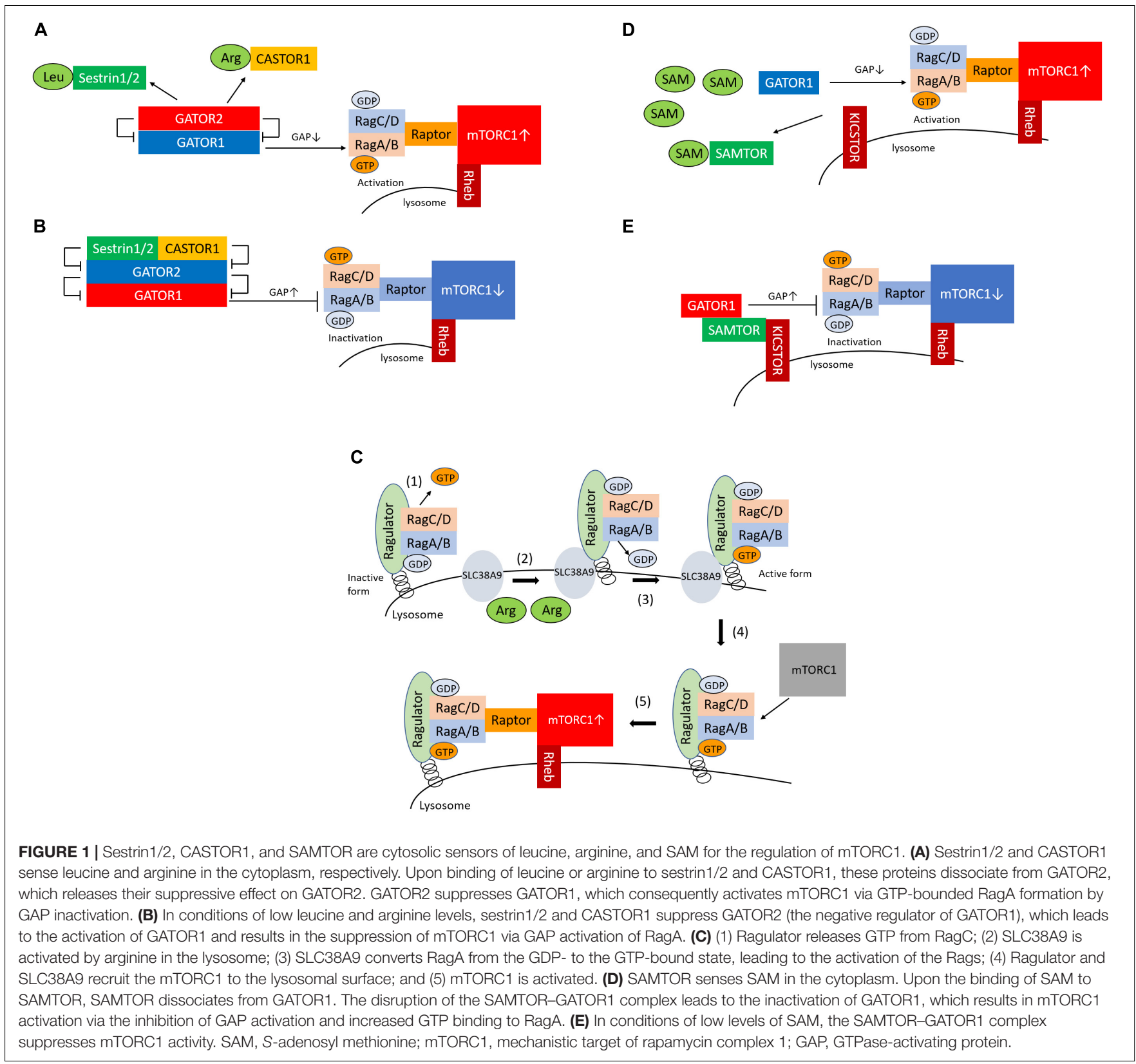

\section{METHIONINE-INDUCED MTORC1 ACTIVATION AND THE ROLE OF SAMTOR AS A SAM SENSOR THAT PROVIDES A LINK TO THE METHIONINE METABOLISM}

The KICSTOR complex is one of the regulators of mTORC1 and comprises kaptin (KPTN), the integrin- $\alpha$ FG-GAP repeatcontaining protein 2 (ITFG2), C12orf66, and seizure threshold 2 (SZT2) (Wolfson et al., 2017). C7orf60 was identified as an interacting protein of GATOR1 and was subsequently renamed SAMTOR (Gu et al., 2017). The overexpression of SAMTOR suppresses mTORC1 activity, which indicates that
SAMTOR is a negative regulator of mTORC1. SAM is converted from methionine, and methionine starvation reduces the concentration of SAM in the cytoplasm. When present of SAM, SAM binds to SAMTOR, which then dissociates from GATOR1 (Figure 1D). The disruption of the SAMTOR-GATOR1 complex leads to the inactivation of GATOR1, which then results in mTORC1 activation through the inhibition of GAP activation and increased binding of GTP to RagA. In contrast, methionine starvation reduces SAM levels below the dissociation constant of the SAM-SAMTOR complex, thus promoting SAMTORGATOR1 binding and, in turn, suppressing mTORC1 activity (Figure 1E). However, loss of SAMTOR activates mTORC1, even in conditions of methionine starvation. In addition, SAMTOR mutants that cannot bind to SAM fail to transmit methionine 

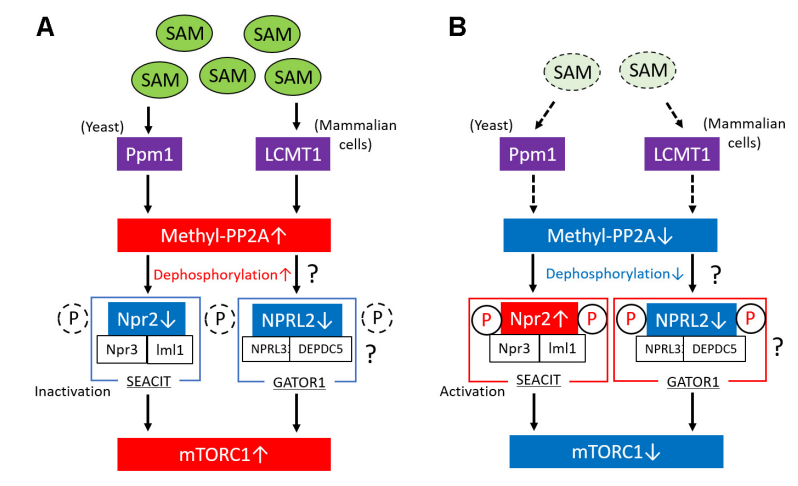

FIGURE 2 | Regulation of mTORC1 via the methylation of PP2A in response to SAM. (A) In conditions of high levels of intracellular SAM in yeast, Ppm1 induces the methylation of the catalytic subunit of PP2A in response to SAM concentration. The activated (methylated) form of PP2A suppresses Npr2 through its dephosphorylation. The complex consisting of Npr2, Npr3, and Iml1 (SEACIT) is a negative regulator of $\mathrm{mTORC1}$; therefore, the suppression of SEACIT via the dephosphorylation of Npr2 results in the activation of mTORC1. In mammalian cells, LCMT1 induces the methylation of the catalytic subunit of PP2A in response to SAM concentration, leading to the activation of mTORC1, possibly through the activation of GATOR1. Moreover, in mammalian cells, PP2A possibly regulates the phosphorylation levels of NPRL2 in response to SAM levels. (B) Lower SAM levels reduce the methylation levels of PP2A in yeast and mammalian cells and promote the activation of Npr2 via its phosphorylation, which results in the suppression of mTORC1 activity. In mammalian cells, PP2A possibly regulates the phosphorylation levels of NPRL2 in response to SAM levels. mTORC1, mechanistic target of rapamycin complex 1; PP2A, phosphatase 2A; SAM, $S$-adenosyl methionine; GAP, GTPase-activating protein; LCMT1, leucine carboxyl methyltransferase 1.

sufficiently to mTORC1, therefore suppressing mTORC1. These results indicate that SAMTOR serves as a SAM sensor in the methionine-mediated mTORC1 activation.

\section{ROLE OF THE INDUCTION OF THE METHYLATION OF PP2A BY SAM IN MTORC1 ACTIVATION}

The study performed by Sutter et al. also showed that methionine regulates the mTORC1 signaling pathway and autophagy through the regulation of the methylation status of phosphatase $2 \mathrm{~A}$ (PP2A) in yeast (Sutter et al., 2013; Laxman et al., 2014). In the presence of high levels of intracellular SAM, Ppm1 induces the methylation of the catalytic subunit of $\mathrm{PP} 2 \mathrm{~A}$ in response to SAM concentration. PP2A is activated by its methylation; thereafter, methylated PP2A can suppress Npr2 through its dephosphorylation, which results in mTORC1 activation and the suppression of autophagy (Figure 2A). The complex consisting of Npr2, Npr3, and Iml1 (NPRL2, NPRL3, and DEPDC5 in mammals, respectively) is termed SEACIT in yeast (GATOR1 in mammals, as described above) (Panchaud et al., 2013) and functions as a negative regulator of $\mathrm{mTORC1}$ via a GAP activity toward the yeast Rag orthologs, that is, Gtr1/2 (Rags family in mammals) (Gao and Kaiser, 2006). Therefore, suppression of SEACIT by the dephosphorylation of Npr2 induced by the activation of $\mathrm{PP} 2 \mathrm{~A}$ results in the activation of mTORC1. In contrast, lower SAM levels in cells reduce the methylation levels of PP2A and promote the phosphorylation of Npr2, which results in the suppression of mTORC1 activity and the induction of autophagy (Figure 2B). In mammalian cells, the methylation of PP2A is catalyzed by a specific $S$-adenosyl methionine (SAM)-dependent methyltransferase, the leucine carboxyl methyltransferase 1 (LCMT1) (Stanevich et al., 2011). Activated PP2A possibly dephosphorylates NPRL2 and results in mTORC1 activation in mammalian cells; however, no report has shown whether PP2A is directly involved in the regulation of the phosphorylation state of NPRL2. Therefore, further studies are necessary to clarify this issue.

We also reported that a low-protein diet ameliorates diabetesinduced kidney injury and that dietary methionine abrogates the beneficial effects of a low-protein diet in diabetic kidneys (Kitada et al., 2020). More specifically, diabetic rats that were fed a low-protein + methionine diet exhibited increased expression of LCMT1 and methyl-PP2A compared with control (standarddiet-fed) and low-protein-diet-fed diabetic rats, which was accompanied by an increase in renal SAM levels. Although the expression of glycine $N$-methyltransferase (Gnmt), which is a SAM-converted enzyme, was decreased in diabetic rat kidneys, changes in renal SAM levels were dependent on the dietary methionine content (Kitada et al., 2020). Consistent with the alteration of LCMT1 and methyl-PP2A, mTORC1 activation and autophagy suppression were observed in standard-diet-fed and low-protein + methionine-fed diabetic rats. Furthermore, we also used cultured human kidney-2 cells to confirm that the administration of SAM-induced methylated PP2A increased the expression of methyl-PP2A and activated mTORC1 (Kitada et al., 2020). However, the involvement of SAM-induced methylated PP2A in mTORC1 activation through NPRL2 and the activation of the negative regulator of mTORC1 by its increased phosphorylation, such as that observed for Npr2 in yeast, remain unknown.

\section{METHIONINE ACTIVATES MTORC1 THROUGH TAS1R1/TAS1R3}

Nelson et al. previously identified a mammalian amino-acid taste receptor, the taste 1 receptor member 1 (TAS1R1)/taste 1 receptor member 3 (TAS1R3) heterodimer, which is a cell-surface G-protein-coupled receptor (Nelson et al., 2002). This receptor broadly functions as an amino-acid sensor that responds to most of the 20 standard amino acids. Upon sensing amino acids, this receptor activates mTORC1 through the activation of phospholipase $\mathrm{C}$, the increase in intracellular calcium, and the activation of the mitogen-activated protein kinase $1 /$ mitogen-activated protein kinase 3 (Wauson et al., 2015). TAS1R1-TAS1R3 is required for the amino-acid-induced mTORC1 localization to the lysosome, which is a necessary step in mTORC1 activation. Several reports have demonstrated that TAS1R1-TAS1R3 may serve as a sensor of extracellular methionine and that it activates mTORC1 in cultured C2C12 cells and bovine epithelial cells (Zhou et al., 2016, 2018). 


\section{DISCUSSION}

In this study, we described the recent findings regarding the mechanism via which methionine induces the activation of mTORC1. mTORC1 may be activated by sensing SAM rather than methionine. A previous report by Obata et al. provided evidence that SAM, rather than methionine, may be the main contributor to the aging process (Obata and Miura, 2015). Those authors showed that increasing SAM catabolism via the action of glycine $\mathrm{N}$-methyltransferase (Gnmt) extends the lifespan in Drosophila. In particular, SAM is upregulated in older flies, even if the transcription of Gnmt is induced in a forkhead box O (FOXO)-dependent manner. However, overexpression of Gnmt suppresses the age-dependent increase in SAM and extends lifespan in Drosophila. In addition, metabolic impairment, such as insulin resistance in obesity, is closely involved in the aging process. A previous report demonstrated that plasma SAM concentrations were related to higher fasting insulin levels, the homeostasis model assessment of insulin resistance, and the tumor necrosis factor $\alpha$ in a cross-sectional study that involved subjects with metabolic syndrome (Lind et al., 2018). Another report also revealed that plasma SAM, and not methionine, is independently related to fat mass and truncal adiposity in a cross-sectional study involving elderly individuals (Elshorbagy et al., 2013); in contrast, overfeeding increases serum SAM in proportion to the fat mass gained (Elshorbagy et al., 2016). Thus, the upregulation of SAM associated with overfeeding or metabolic dysfunction may be involved in whole-body metabolic impairment. These data indicate that increased levels of SAM in the process of methionine metabolism may be related to the stimulation of aging and metabolic impairment, including insulin resistance, which is particularly associated with obesity. Previous reports

\section{REFERENCES}

Abu-Remaileh, M., Wyant, G. A., Kim, C., Laqtom, N. N., Abbasi, M., Chan, S. H., et al. (2017). Lysosomal metabolomics reveals V-ATPase- and mTORdependent regulation of amino acid efflux from lysosomes. Science 358, 807813. doi: $10.1126 /$ science.aan6298

Anandapadamanaban, M., Masson, G. R., Perisic, O., Berndt, A., Kaufman, J., Johnson, C. M., et al. (2019). Architecture of human Rag GTPase heterodimers and their complex with mTORC1. Science 366, 203-210. doi: 10.1126/science. aax3939

Anisimov, V. N., Zabezhinski, M. A., Popovich, I. G., Piskunova, T. S., Semenchenko, A. V., Tyndyk, M. L., et al. (2011). Rapamycin increases lifespan and inhibits spontaneous tumorigenesis in inbred female mice. Cell Cycle 10, 4230-4236. doi: 10.4161/cc.10.24.18486

Bar-Peled, L., Chantranupong, L., Cherniack, A. D., Chen, W. W., Ottina, K. A., Grabiner, B. C., et al. (2013). A tumor suppressor complex with GAP activity for the Rag GTPases that signal amino acid sufficiency to mTORC1. Science 340, 1100-1106. doi: 10.1126/science.1232044

Chantranupong, L., Scaria, S. M., Saxton, R. A., Gygi, M. P., Shen, K., Wyant, G. A., et al. (2016). The CASTOR proteins are arginine sensors for the mTORC1 pathway. Cell 165, 153-164.

Chantranupong, L., Wolfson, R. L., Orozco, J. M., Saxton, R. A., Scaria, S. M., Bar-Peled, L., et al. (2014). The sestrins interact with GATOR2 to negatively regulate the amino-acid-sensing pathway upstream of mTORC1. Cell Rep. 9, 1-8. doi: 10.1016/j.celrep.2014.09.014 have shown that dietary methionine restriction extends the lifespan or improves cardiometabolic health (Orentreich et al., 1993; Miller et al., 2005; Hasek et al., 2010; Plaisance et al., 2011; Johnson and Johnson, 2014; Lee et al., 2014; Stone et al., 2014). The effect of methionine restriction on lifespan extension or cardiometabolic health may be exerted through multiple mechanisms, including antioxidative stress, the production of hydroxy sulfates, the downregulation of $\mathrm{GH} /$ insulin growth factor 1 signaling, the production of fibroblast growth factor 21 , the suppression of mTORC1, and the induction of autophagy (Kitada et al., 2019). Among them, the suppression of mTORC1 is induced by decreasing SAM levels. Therefore, the regulation of SAM levels and sensing of SAM in the cytoplasm may be key factors in the mechanism of lifespan extension, which may be mediated by the regulation of mTORC1. Because the selective suppression of mTORC1 induced by SAM may be a therapeutic target for aging, metabolic impairment, or aging-related disease, further studies are necessary to address these issues.

\section{AUTHOR CONTRIBUTIONS}

MK designed the manuscript, the guarantor of this work, and wrote and edited the manuscript. JX, YO, IM, and DK contributed to the discussion. All authors contributed to the article and approved the submitted version.

\section{ACKNOWLEDGMENTS}

This study was supported in part by the Japan-China Sasakawa Medical Fellowship to JX.

Colman, R. J., Anderson, R. M., Johnson, S. C., Kastman, E. K., Kosmatka, K. J., Beasley, T. M., et al. (2009). Caloric restriction delays disease onset and mortality in rhesus monkeys. Science 325, 201-204. doi: 10.1126/science. 1173635

Cummings, N. E., Williams, E. M., Kasza, I., Konon, E. N., Schaid, M. D., Schmidt, B. A., et al. (2018). Restoration of metabolic health by decreased consumption of branched-chain amino acids. J. Physiol. 596, 623-645.

Efeyan, A., Comb, W. C., and Sabatini, D. M. (2015). Nutrient-sensing mechanisms and pathways. Nature 517, 302-310. doi: 10.1038/nature14190

Elshorbagy, A. K., Jerneren, F., Samocha-Bonet, D., Refsum, H., and Heilbronn, L. K. (2016). Serum S-adenosylmethionine, but not methionine, increases in response to overfeeding in humans. Nutr Diabetes. 6:e192. doi: 10.1038/nutd. 2015.44

Elshorbagy, A. K., Nijpels, G., Valdivia-Garcia, M., Stehouwer, C. D., Ocke, M., Refsum, H., et al. (2013). S-adenosylmethionine is associated with fat mass and truncal adiposity in older adults. J. Nutr. 143, 1982-1988. doi: 10.3945/jn.113. 179192

Fontana, L., Cummings, N. E., Arriola Apelo, S. I., Neuman, J. C., Kasza, I., Schmidt, B. A., et al. (2016). Decreased consumption of branched-chain amino acids improves metabolic health. Cell Rep. 16, 520-530.

Fontana, L., Partridge, L., and Longo, V. D. (2010). Extending healthy life spanfrom yeast to humans. Science 328, 321-326. doi: 10.1126/science.1172539

Gao, M., and Kaiser, C. (2006). A. A conserved GTPase-containing complex is required for intracellular sorting of the general amino-acid permease in yeast. Nat. Cell Biol. 8, 657-667. doi: 10.1038/ncb1419 
Gu, X., Orozco, J. M., Saxton, R. A., Condon, K. J., Liu, G. Y., Krawczyk, P. A., et al. (2017). SAMTOR is an S-adenosylmethionine sensor for the mTORC1 pathway. Science 358, 813-818.

Harrison, D. E., Strong, R., Sharp, Z. D., Nelson, J. F., Astle, C. M., Flurkey, K., et al. (2009). Rapamycin fed late in life extends lifespan in genetically heterogeneous mice. Nature 460, 392-395. doi: 10.1038/nature 08221

Hasek, B. E., Stewart, L. K., Henagan, T. M., Boudreau, A., Lenard, N. R., Black, C., et al. (2010). Dietary methionine restriction enhances metabolic flexibility and increases uncoupled respiration in both fed and fasted states. Am. J. Physiol. Regul. Integr. Comp. Physiol. 299, R728-R739.

Johnson, J. E., and Johnson, F. B. (2014). Methionine restriction activates the retrograde response and confers both stress tolerance and lifespan extension to yeast, mouse and human cells. PLoS One 9:e97729. doi: 10.1371/journal.pone. 0097729

Kim, E., Goraksha-Hicks, P., Li, L., Neufeld, T. P., and Guan, K. L. (2008). Regulation of TORC1 by Rag GTPases in nutrient response. Nat. Cell Biol. 10, 935-945. doi: 10.1038/ncb1753

Kim, J., and Guan, K. L. (2019). mTOR as a central hub of nutrient signalling and cell growth. Nat. Cell Biol. 21, 63-71. doi: 10.1038/s41556-0180205-1

Kitada, M., Ogura, Y., Monno, I., and Koya, D. (2019). The impact of dietary protein intake on longevity and metabolic health. EBioMedicine 43, 632-640. doi: 10.1016/j.ebiom.2019.04.005

Kitada, M., Ogura, Y., Monno, I., Xu, J., and Koya, D. (2020). Methionine abrogates the renoprotective effect of a low-protein diet against diabetic kidney disease in obese rats with type 2 diabetes. Aging 12, 4489-4505. doi: 10.18632/aging. 102902

Laxman, S., Sutter, B. M., and Tu, B. P. (2014). Methionine is a signal of amino acid sufficiency that inhibits autophagy through the methylation of PP2A. Autophagy 10, 386-387. doi: 10.4161/auto.27485

Lee, B. C., Kaya, A., and Gladyshev, V. N. (2016). Methionine restriction and life-span control. Ann. N. Y. Acad. Sci. 1363, 116-124. doi: 10.1111/nyas. 12973

Lee, B. C., Kaya, A., Ma, S., Kim, G., Gerashchenko, M. V., Yim, S. H., et al. (2014). Methionine restriction extends lifespan of Drosophila melanogaster under conditions of low amino-acid status. Nat Commun. 5:3592. doi: 10.1038/ ncomms 4592

Levine, M. E., Suarez, J. A., Brandhorst, S., Balasubramanian, P., Cheng, C. W., Madia, F., et al. (2014). Low protein intake is associated with a major reduction in IGF-1, cancer, and overall mortality in the 65 and younger but not older population. Cell Metab. 19, 407-417. doi: 10.1016/j.cmet.2014.02. 006

Lind, M. V., Lauritzen, L., Vestergaard, H., Hansen, T., Pedersen, O., Kristensen, M., et al. (2018). One-carbon metabolism markers are associated with cardiometabolic risk factors. Nutr. Metab. Cardiovasc. Dis. 28, 402-410. doi: 10.1016/j.numecd.2018.01.005

Mattison, J. A., Colman, R. J., Beasley, T. M., Allison, D. B., Kemnitz, J. W., Roth, G. S., et al. (2017). Caloric restriction improves health and survival of rhesus monkeys. Nat. Commun. 8:14063. doi: 10.1038/ncomms14063

Mattison, J. A., Roth, G. S., Beasley, T. M., Tilmont, E. M., Handy, A. M., Herbert, R. L., et al. (2012). Impact of caloric restriction on health and survival in rhesus monkeys from the NIA study. Nature 489, 318-321.

Miller, R. A., Buehner, G., Chang, Y., Harper, J. M., Sigler, R., SmithWheelock, M., et al. (2005). Methionine-deficient diet extends mouse lifespan, slows immune and lens aging, alters glucose, T4, IGF-I and insulin levels, and increases hepatocyte MIF levels and stress resistance. Aging Cell 4, 119-125. doi: 10.1111/j.1474-9726.2005. 00152.x

Miller, R. A., Harrison, D. E., Astle, C. M., Fernandez, E., Flurkey, K., Han, M., et al. (2014). Rapamycin-mediated lifespan increase in mice is dose and sex dependent and metabolically distinct from dietary restriction. Aging Cell 13, 468-477. doi: 10.1111/acel.12194

Mizushima, N., Levine, B., Cuervo, A. M., and Klionsky, D. J. (2008). Autophagy fights disease through cellular self-digestion. Nature 451, 1069-1075. doi: 10. 1038/nature06639
Nakagawa, S., Lagisz, M., Hector, K. L., and Spencer, H. G. (2012). Comparative and meta-analytic insights into life extension via dietary restriction. Aging Cell 11, 401-409. doi: 10.1111/j.1474-9726.2012.00798.x

Nelson, G., Chandrashekar, J., Hoon, M. A., Feng, L., Zhao, G., Ryba, N. J. P., et al. (2002). An amino-acid taste receptor. Nature 416, 199-202.

Obata, F., and Miura, M. (2015). Enhancing S-adenosyl-methionine catabolism extends Drosophila lifespan. Nat Commun. 6:8332. doi: 10.1038/ncomms9332

Orentreich, N., Matias, J. R., DeFelice, A., and Zimmerman, J. A. (1993). Low methionine ingestion by rats extends life span. J. Nutr. 123, 269-274.

Panchaud, N., Péli-Gulli, M.-P., and De Virgilio, C. (2013). Amino acid deprivation inhibits TORC1 through a GTPase-activating protein complex for the Rag family GTPase Gtr1. Sci. Signal. 6:ra42. doi: 10.1126/scisignal.200 4112

Parmigiani, A., Nourbakhsh, A., Ding, B., Wang, W., Kim, Y. C., Akopiants, K., et al. (2014). Sestrins inhibit mTORC1 kinase activation through the GATOR complex. Cell Rep. 9, 1281-1291. doi: 10.1016/j.celrep.2014. 10.019

Plaisance, E. P., Greenway, F. L., Boudreau, A., Hill, K. L., Johnson, W. D., Gettys, T. W., et al. (2011). Dietary methionine restriction increases fat oxidation in obese adults with metabolic syndrome. J. Clin. Endocrinol. Metab. 96, E836E840.

Sancak, Y., Peterson, T. R., Shaul, Y. D., Lindquist, R. A., Thoreen, C. C., BarPeled, L., et al. (2008). The Rag GTPases bind Raptor and mediate amino acid signaling to mTORC1. Science 320, 1496-1501. doi: 10.1126/science.115 7535

Saxton, R. A., Chantranupong, L., Knockenhauer, K. E., Schwartz, T. U., and Sabatini, D. M. (2016a). Mechanism of arginine sensing by CASTOR1 upstream of mTORC1. Nature 536, 229-233. doi: 10.1038/nature19079

Saxton, R. A., Knockenhauer, K. E., Wolfson, R. L., Chantranupong, L., Pacold, M. E., Wang, T., et al. (2016b). Structural basis for leucine sensing by the sestrin2-mTORC1 pathway. Science 351, 53-58. doi: 10.1126/science.aad2087

Shen, K., Huang, R. K., Brignole, E. J., Condon, K. J., Valenstein, M. L., Chantranuponget, L., et al. (2018). Architecture of the human GATOR1 and GATOR1-Rag GTPases complexes. Nature 556, 64-69.

Shen, K., and Sabatini, D. M. (2018). Ragulator and SLC38A9 activate the Rag GTPases through noncanonical GEF mechanisms. Proc. Natl. Acad. Sci. U.S.A. 115, 9545-9550. doi: 10.1073/pnas.18117 27115

Simpson, S. J., Le Couteur, D. G., James, D. E., Georg, J., Gunton, J. E., SolonBiet, S. M., et al. (2017). The Geometric Framework for Nutrition as a tool in precision medicine. Nutr. Healthy Aging 4, 217-226. doi: 10.3233/nha-17 0027

Solon-Biet, S. M., McMahon, A. C., Ballard, J. W., Ruohonen, K., Wu, L. E., Cogger, V. C., et al. (2014). The ratio of macronutrients, not caloric intake, dictates cardiometabolic health, aging, and longevity in ad libitum-fed mice. Cell Metab. 19, 418-430. doi: 10.1016/j.cmet.2014.02.009

Stanevich, V., Jiang, L., Satyshur, K. A., Li, Y., Jeffrey, P. D., Li, Z., et al. (2011). The structural basis for tight control of PP2A methylation and function by LCMT-1. Mol. Cell. 41, 331-342. doi: 10.1016/j.molcel.2010.12.030

Stone, K. P., Wanders, D., Orgeron, M., Cortez, C. C., and Gettys, T. W. (2014). Mechanisms of increased in vivo insulin sensitivity by dietary methionine restriction in mice. Diabetes Metab. Res. Rev. 63, 3721-3733. doi: 10.2337/db140464

Sutter, B. M., Wu, X., Laxman, S., and Tu, B. P. (2013). Methionine inhibits autophagy and promotes growth by inducing the SAM-responsive methylation of PP2A. Cell 154, 403-415. doi: 10.1016/j.cell.2013.06.041

Wauson, E. M., Guerra, M. L., Dyachok, J., McGlynn, K., Giles, J., Ross, E. M., et al. (2015). Differential regulation of ERK1/2 and mTORC1 through T1R1/T1R3 in MIN6 cells. Mol. Endocrinol. 29, 1114-1122. doi: 10.1210/me.20141181

Wilkinson, J. E., Burmeister, L., Brooks, S. V., Chan, C. C., Friedline, S., Harrison, D. E., et al. (2012). Rapamycin slows aging in mice. Aging Cell 11, 675-682. doi: 10.1111/j.1474-9726.2012.00832.x

Wolfson, R. L., Chantranupong, L., Saxton, R. A., Shen, K., Scaria, S. M., Cantor, J. R., et al. (2016). Sestrin2 is a leucine sensor for the mTORC1 pathway. Science 351, 43-48. doi: 10.1126/science.aab2674 
Wolfson, R. L., Chantranupong, L., Wyant, G. A., Gu, X., Orozco, J. M., Shen, K., et al. (2017). KICSTOR recruits GATOR1 to the lysosome and is necessary for nutrients to regulate mTORC1. Nature 543, 438-442. doi: 10.1038/nature21423

Wong, S. Q., Kumar, A. V., Mills, J., and Lapierre, L. R. (2020). Autophagy in aging and longevity. Hum. Genet. 139, 277-290.

Wyant, G. A., Abu-Remaileh, M., Wolfson, R. L., Chen, W. W., Freinkman, E., Danai, L. V., et al. (2017). mTORC1 activator SLC38A9 is required to efflux essential amino acids from lysosomes and use protein as a nutrient. Cell 171, 642-654.

Zhang, Y., Bokov, A., Gelfond, J., Soto, V., Ikeno, Y., Hubbard, G., et al. (2014). Rapamycin extends life and health in C57BL/6 mice. J. Gerontol. A Biol. Sci. Med. Sci. 69, 119-130. doi: 10.1093/gerona/glt056

Zhou, Y., Ren, J., Song, T., Peng, J., and Wei, H. (2016). Methionine regulates mTORC1 via the T1R1/T1R3-PLCbeta-Ca2+-ERK1/2 signal transduction process in C2C12 cells. Int. J. Mol. Sci. 17, 1684. doi: 10.3390/ijms17101684

Zhou, Y., Zhou, Z., Peng, J., and Loor, J. J. (2018). Methionine and valine activate the mammalian target of rapamycin complex 1 pathway through heterodimeric amino acid taste receptor (TAS1R1/TAS1R3) and intracellular Ca $2+$ in bovine mammary epithelial cells. J. Dairy Sci. 101, 11354-11363. doi: 10.3168/jds. 2018-14461
Zoncu, R., Bar-Peled, L., Efeyan, L., Wang, S., Sancak, Y., and Sabatinil, D. M. (2011). mTORC1 senses lysosomal amino acids through an inside-out mechanism that requires the vacuolar $\mathrm{H}^{+}$-ATPase. Science 334, 678-683. doi: 10.1126/science. 1207056

Conflict of Interest: Boehringer Ingelheim, Mitsubishi Tanabe Pharma, Kyowa Kirin, Taisho Pharmaceutical Co., Ltd., and Ono Pharmaceutical Co., Ltd. contributed to establishing the Division of Anticipatory Molecular Food Science and Technology.

The authors declare that the research was conducted in the absence of any commercial or financial relationships that could be construed as a potential conflict of interest.

Copyright (C) 2020 Kitada, Xu, Ogura, Monno and Koya. This is an open-access article distributed under the terms of the Creative Commons Attribution License (CC BY). The use, distribution or reproduction in other forums is permitted, provided the original author(s) and the copyright owner(s) are credited and that the original publication in this journal is cited, in accordance with accepted academic practice. No use, distribution or reproduction is permitted which does not comply with these terms. 